\title{
Calcificación simétrica y bilateral de ganglios basales. Serie de casos y revisión de la literatura
}

\author{
Amado Jiménez-Ruiz, ${ }^{1}$ Omar Cárdenas-Sáenz ${ }^{1}$ y José Luis Ruiz-Sandova² \\ ${ }^{1}$ Medicina Interna; ${ }^{2}$ Neurología.Hospital Civil de Guadalajara Fray Antonio Alcalde, Guadalajara, Jalisco, México
}

\section{Resumen}

Introducción: La calcificación bilateral y simétrica de los ganglios basales es un hallazgo infrecuente que a veces no ocasiona síntomas. Su prevalencia aumenta con la edad y el sitio más afectado es el globo pálido. Reporte de casos: Se describe una serie de siete casos con diagnóstico clínico y por imagen de calcificación de ganglios basales, atendidos entre 2012 y 2016 en el Servicio de Medicina Interna del Hospital Civil de Guadalajara Fray Antonio Alcalde. Las manifestaciones clínicas más comunes fueron alteración del estado de alerta, cefalea y crisis convulsivas. Se identificó un caso con trastornos del movimiento; no hubo casos con demencia o tetania. Conclusión: La calcificación de los ganglios puede estar relacionada con cambios neurodegenerativos por la edad, pero puede ser la manifestación inicial de una variedad de patologías sistémicas, incluyendo trastornos del metabolismo del calcio, intoxicación por diversos agentes, enfermedades autoinmunes y genéticas. Se debe hacer la correlación de los hallazgos de imagen típicos con manifestaciones clínicas y resultados de laboratorio para llegar a un dictamen definitivo.

PALABRAS CLAVE: Calcificación de ganglios basales. Síndrome de Fahr. Hipoparatiroidismo. Síndrome MELAS.

\begin{abstract}
Introduction: Symmetric, bilateral basal ganglia calcification is rare finding that sometimes occurs asymptomatically. Its prevalence increases with age, and the most affected site is the globus pallidus. Report of cases: $A$ series of seven cases with clinical and imaging diagnosis of basal ganglia calcification, recorded during the 2012 to 2016 period at the Department of Internal Medicine of the Hospital Civil de Guadalajara "Fray Antonio Alcalde, is presented. Most common clinical presentation was with altered alertness, headache and seizures. There was one case with movement disorders; there were no cases identified with dementia or tetany. Conclusion: Ganglia calcification can be associated with age-related neurodegenerative changes, but it can be an initial manifestation of a variety of systemic pathologies, including disorders of the calcium metabolism, intoxication by different agents, and autoimmune and genetic diseases. Correlation of typical imaging findings with clinical manifestations and laboratory results should be established to reach a definitive judgment.
\end{abstract}

KEY WORDS: Basal ganglia calcification. Fahr's syndrome. Hypoparathyroidism. MELAS syndrome.

Fecha de recepción: 06-02-2016

Fecha de aceptación: 26-04-2016

DOI://dx.doi.org/10.24875/GMM.18002406
Gac Med Mex. 2018;154:258-262

Disponible en PubMed www.gacetamedicademexico.com 


\section{Introducción}

La calcificación de ganglios basales es un hallazgo inespecífico encontrado en $1 \%$ de todas las tomografías de cráneo realizadas; ${ }^{1}$ para su estudio se categoriza en fisiológica y patológica. La primera se asociación envejecimiento y la segunda con trastornos metabólicos, autoinmunes o enfermedades neurodegenerativas primarias, sobre todo en pacientes jóvenes. Las causas más comunes incluyen los trastornos en el metabolismo del calcio, especialmente hipoparatiroidismo y pseudohipoparatiroidismo. ${ }^{2}$ Sin embargo, también podemos encontrarla debido a exposición a ciertos tóxicos (plomo, monóxido de carbono), asociada a radiación intracraneal y enfermedades neurodegenerativas genéticas. La calcificación de ganglios basales idiopática o enfermedad de Fahr es una entidad rara que puede tener un patrón de herencia específico o presentarse de forma esporádica.

En esta investigación se describen siete casos que ingresaron al Servicio de Medicina Interna del Hospital Civil de Guadalajara Fray Antonio Alcalde, en un periodo de cuatro años, con diagnóstico establecido por imagenología.

\section{Métodos}

Se revisaron los expedientes clínicos de pacientes con diagnóstico de calcificación de ganglios basales establecido por los hallazgos en una tomografía axial computarizada de cráneo, atendidos entre 2012 y 2016. Se consideraron los siguientes criterios de inclusión:

- Hallazgos en tomografía axial computarizada compatibles con calcificación de ganglios basales.

- Contar con diagnóstico etiológico por exámenes paraclínicos.

Se excluyeron los casos con estudios incompletos al ingreso, por lo tanto, con diagnóstico no concluyente.

\section{Resultados}

Los pacientes con diagnóstico de calcificación de ganglios basales por imagen en tomografía axial computarizada fueron siete, dos con calcificación cerebelosa y subcortical concomitante; tres de los siete eran del sexo masculino; la edad promedio de presentación fue de 38 años.

Las manifestaciones clínicas más comunes fueron alteración del estado de alerta, cefalea y crisis convulsivas.
Las manifestaciones clínicas, hallazgos en la tomografía, exámenes de laboratorio y diagnostico etiológico se describen en la Tabla 1. En la Tabla 2 se detallan las alteraciones radiológicas y clínicas más comunes. Por último, en la Tabla 3 se listan los Hubo un caso con trastornos del movimiento; ninguno presentó demencia o tetania. diagnósticos diferenciales según la etiología.

\section{Discusión}

Junto con el cerebelo, el tálamo motor y la corteza frontal constituyen un complejo sistema que garantiza la organización y ejecución de patrones normales de movimiento. $^{3}$

La calcificación simétrica y bilateral ha sido descrita en asociación con diversos trastornos: tóxicos (por monóxido de carbono o plomo), metabólicos del calcio (hipoparatiroidismo, hiperparatiroidismo, pseudohipoparatiroidismo), encefalopatía anoxoisquémica, enfermedades autoinmunes (lupus eritematoso sistémico) y genéticas neurodegenerativas (síndrome MELAS, enfermedad de Cockayne, enfermedad de Hallervorden-Spatz). Cuando no hay una causa identificable se denomina enfermedad de Fahr. ${ }^{4,5}$

Los ganglios calcificados generalmente se detectan de manera incidental en una tomografía computarizada. ${ }^{6}$ Las calcificaciones intracraneales fisiológicas se observan en aproximadamente 0.3 a $1.5 \%$ de los casos; se identifican de manera incidental por neuroimagen y suele ser asintomáticas. En una serie de casos, durante 2012 Abdelrahman revisó de manera retrospectiva 1040 tomografías de cráneo, 552 correspondieron a hombres; encontró calcificación de los ganglios basales en $1.25 \%$ (0.72\% en hombres y $1.85 \%$ en mujeres; $\mathrm{RM}=2.57$ [0.72-9.98], RR=2.55 [0.79-8.21], $p=0.1){ }^{6.7}$

Al ser parte de las entidades mencionadas, la mayoría de los casos presenta síntomas que varían desde trastornos del movimiento (parkinsonismo, corea, atetosis), alteración del estado de alerta, cefalea y crisis convulsivas hasta síndromes como el de la motoneurona superior y cuadros neuropsiquiátricos. ${ }^{8,9}$

La causa por la cual los pacientes descritos presentaban calcificación en los ganglios basales fue distinta en cada uno; según la historia clínica y la exploración física inicial se realizó protocolo específico para llegar a un diagnóstico definitivo.

El síndrome MELAS es causado por diferentes mutaciones puntuales del ADN mitocondrial, cuya herencia es materna; la más frecuente (en 80 a $85 \%$ ) es la que se localiza en posición A3243G del ARNt DEL ADN materno. La primera descripción del síndrome 
Tabla 1. Presentación clínica, hallazgos en la tomografía, exámenes de laboratorio y diagnostico etiológico en siete pacientes con calcificación de ganglios basales

\begin{tabular}{|c|c|c|c|c|c|c|c|c|}
\hline Caso & Sexo & Edad & $\begin{array}{l}\text { Calcificación } \\
\text { de ganglios } \\
\text { basales }\end{array}$ & $\begin{array}{l}\text { Calcificaciones } \\
\text { cerebelosas }\end{array}$ & $\begin{array}{l}\text { Calcificaciones } \\
\text { subcorticales }\end{array}$ & $\begin{array}{l}\text { Presentación } \\
\text { clínica }\end{array}$ & Hallazgos & Diagnóstico etiológico \\
\hline 1 & M & 51 & Presentes & Presentes & Presentes & $\begin{array}{l}\text { Alteración del } \\
\text { estado de alerta, } \\
\text { cefalea, crisis } \\
\text { convulsivas, } \\
\text { hemiparesia }\end{array}$ & $\begin{array}{l}\text { Hiperlactatemia, } \\
\text { diabetes mellitus, } \\
\text { hipoacusia, biopsia } \\
\text { de músculo con fibras } \\
\text { rojas rasgadas en } \\
\text { tinción de Gomori }\end{array}$ & MELAS \\
\hline 2 & M & 46 & Presentes & Ausentes & Presentes & $\begin{array}{l}\text { Alteración del } \\
\text { estado de altera, } \\
\text { crisis convulsivas }\end{array}$ & $\begin{array}{l}\text { Calcio } 3.6 \mathrm{mg} / \mathrm{dL} \\
(8.5-10.1), \\
\text { Fosfato } 8.3 \mathrm{mg} / \mathrm{dL} \\
(2.4-4.5) \\
\text { PTH } 126 \mathrm{pg} / \mathrm{mL} \\
(4.6-58.1)\end{array}$ & Pseudohipoparatirodismo \\
\hline 3 & M & 62 & Presentes & Presentes & Presentes & $\begin{array}{l}\text { Crisis convulsivas, } \\
\text { catarata bilateral }\end{array}$ & $\begin{array}{l}\text { Calcio } 6.1 \mathrm{mg} / \mathrm{dL} \\
(8.5-10.1) \\
\text { Fosfato } 5 \mathrm{mg} / \mathrm{dL} \\
(2.4-4.5) \\
\text { PTH } 1 \mathrm{pg} / \mathrm{mL} \\
(4.6-58.1)\end{array}$ & Hipoparatiroidismo \\
\hline 4 & F & 34 & Presentes & Ausentes & Ausentes & $\begin{array}{l}\text { Hemiparesia y } \\
\text { vértigo }\end{array}$ & $\begin{array}{l}\text { Calcio y fosfato } \\
\text { normales } \\
\text { PTH normal }\end{array}$ & Fahr \\
\hline 5 & F & 17 & Presentes & Ausentes & Ausentes & $\begin{array}{l}\text { Alteración del } \\
\text { estado de altera }\end{array}$ & $\begin{array}{l}\text { Anticuerpos } \\
\text { antinucleares } \\
\text { positivos }\end{array}$ & $\begin{array}{l}\text { Lupus Eritematoso } \\
\text { Sistémico }\end{array}$ \\
\hline 6 & F & 20 & Presentes & Ausentes & Ausentes & Crisis convulsivas & $\begin{array}{l}\text { Hiperlactatemia, } \\
\text { diabetes mellitus, } \\
\text { hipoacusia, biopsia } \\
\text { de músculo con fibras } \\
\text { rojas rasgadas en } \\
\text { tinción de Gomori }\end{array}$ & MELAS \\
\hline 7 & $\mathrm{~F}$ & 42 & Presentes & Ausentes & Ausentes & $\begin{array}{l}\text { Alteración del } \\
\text { estado de alerta }\end{array}$ & $\begin{array}{l}\text { Hiperlactatemia, } \\
\text { diabetes mellitus, } \\
\text { hipoacusia, biopsia } \\
\text { de músculo con fibras } \\
\text { rojas rasgadas en } \\
\text { tinción de Gomori }\end{array}$ & MELAS \\
\hline
\end{tabular}

Tabla 2. Alteraciones radiológicas y clínicas más comunes en siete pacientes con calcificación de ganglios basales

\begin{tabular}{llc}
\hline & $\mathbf{n}$ & $\%$ \\
\hline Calcificaciones cerebelosas & 2 & 28 \\
Calcificaciones subcorticales & 3 & 2 \\
Alteración del estado de alerta & 4 & 57 \\
Crisis convulsivas & 4 & 57 \\
Cefalea & 1 & 14 \\
Trastornos motores & 2 & 28 \\
Parkinsonismo & 1 & 14 \\
\hline
\end{tabular}

MELAS fue realizada por Pavlakis et al. en 1984, quienes lo definen como uno de los síndromes mitocondriales multisistémicos relacionados con accidentes cerebrovasculares antes de los 40 años, encefalopatía caracterizada por crisis epilépticas focales o generalizadas, acidosis láctica y fibras rojas rasgadas en la biopsia muscular. Es frecuente el hallazgo de diabetes mellitus, así como hipoacusia bilateral. Aproximadamente $30 \%$ de los pacientes con MELAS presenta calcificación de ganglios basales. ${ }^{10-12}$

En la Figura 1 se puede apreciar un ejemplo del síndrome de MELAS. 
Tabla 3. Principales causas de calcificación de ganglios basales

\begin{tabular}{llll}
\hline Etiología & Ejemplo & Etiología & Ejemplo \\
\hline Idiopática & - Envejecimiento & Tóxico & - Monóxido de carbono \\
& - Enfermedad de Fahr & & - Plomo \\
& & & - Microangiopatía por mineralización \\
Metabólica & - Hipoparatiroidismo & Misceláneos & - Lupus eritematoso sistémico \\
& - Pseudohipoparatiroidismo & & - Quimioterapia/radioterapia \\
Hereditaria & - Enfermedades mitocondriales (MELAS/MERRF) & & \\
& - Síndrome Aicardi-Goutières & & \\
& - Síndrome de Cockayne & & \\
& - Síndrome de Down & & \\
\hline
\end{tabular}

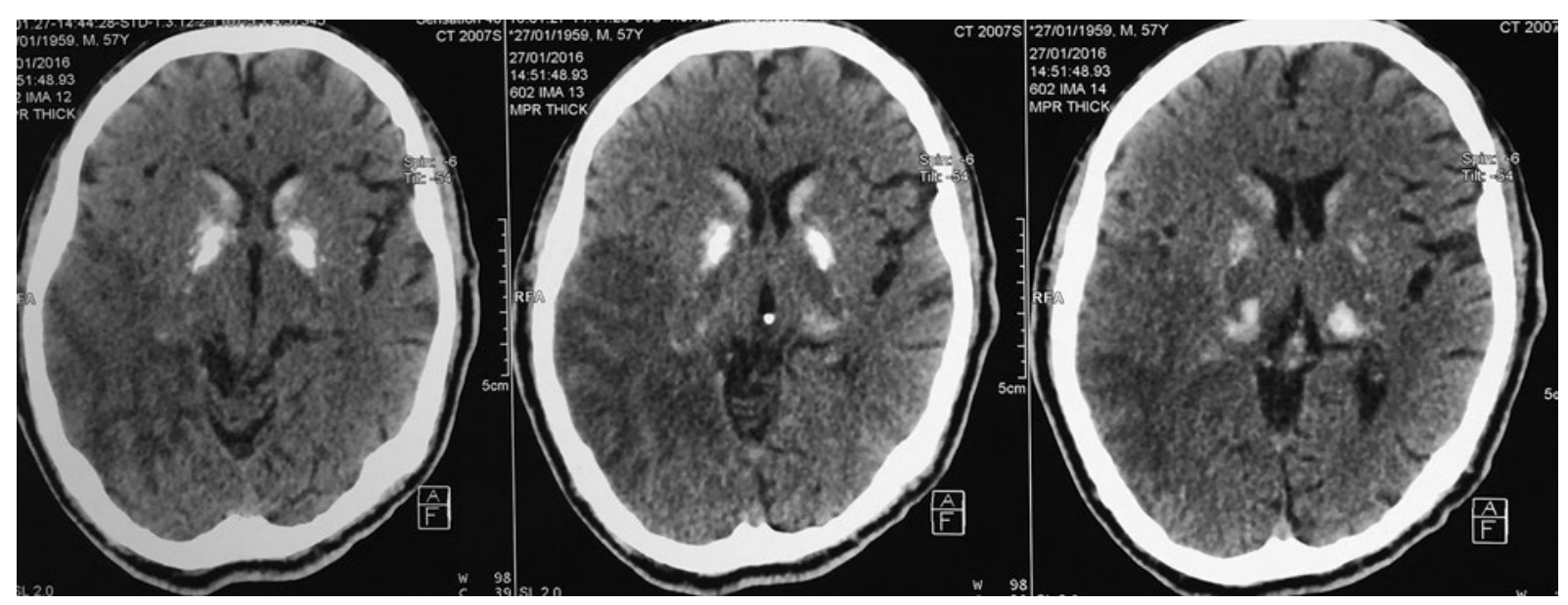

Figura 1. Caso 1. Síndrome de MELAS. Calcificaciones bilaterales en ganglios basales e hipodensidad parieto-occipital consistente con stroke like por resonancia magnética.

El hipoparatiroidismo es una deficiencia endocrina caracterizada por niveles bajos de calcio sérico, niveles elevados de fósforo y ausencia o niveles inapropiadamente bajos de hormona paratiroidea (PTH) sérica. Puede acompañarse de convulsiones secundarias a niveles bajos de calcio o a la calcificación simétrica y bilateral de diversas estructuras cerebrales, como los ganglios basales, subcorticales o cerebelares. ${ }^{13}$

El pseudohipoparatiroidismo es un trastorno hereditario heterogéneo poco frecuente, caracterizado por hipocalcemia-hiperfosfatemia con PTH plasmática elevada en ausencia de daño renal. Fue descrito por primera vez en 1942 por Albright; en algunas series de casos se presentaron calcificaciones extraesqueléticas hasta en $84 \%$ de los pacientes, incluidas las de ganglios basales. ${ }^{13-15}$

En las Figuras 2 y 3 podemos ver ejemplos de los hallazgos tomográficos en el pseudohipoparatiroidismo e hipoparatiroidismo.

La enfermedad de Fahr consiste en calcificaciones simétricas, localizadas principalmente en los ganglios basales y el cerebelo, asociada con trastornos neurológicos variados, entre los cuales los más frecuentes son los del movimiento de tipo extrapiramidal (con predominio de parkinsonismo, aunque también se encuentra coreoatetosis, distonía, temblor, discinesias orofaciales y corea paroxística), demencia y alteraciones psiquiátricas; en pocas ocasiones se relacionan alteraciones sensoriales. El mecanismo fisiopatológico reside en el depósito de calcio y otros minerales en el globo pálido, putamen, corona radiada, tálamo, núcleos dentados cerebelosos y sustancia blanca hemisférica y cerebelosa; estos depósitos cálcicos se forman alrededor de un nido de polisacárido que se produce primariamente en las paredes de arteriolas y capilares. Para su diagnóstico es necesaria la demostración de familiares de primera línea afectados y la exclusión de los trastornos mencionados. ${ }^{16,17}$

El lupus eritematoso sistémico es una enfermedad autoinmune común que afecta a mujeres jóvenes y se presenta con sintomatología articular, pleuropulmonar, cutánea, hematológica, renal y neurológica. En cuanto a su asociación con la calcificación de ganglios basales, el mecanismo fisiopatológico no se conoce con exactitud, si bien diversas teorías intentan explicar su aparición..$^{18,19}$ 


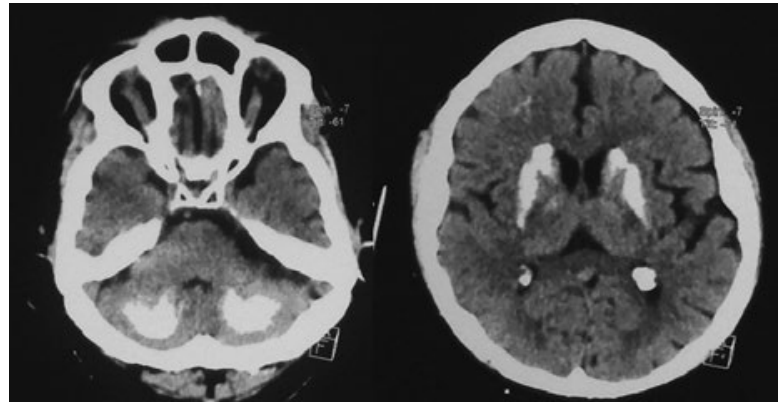

Figura 2. Caso 3. Pseudohipoparatiroidismo. Calcificaciones bilaterales simétricas en ganglios basales y hemisferios cerebelosos.

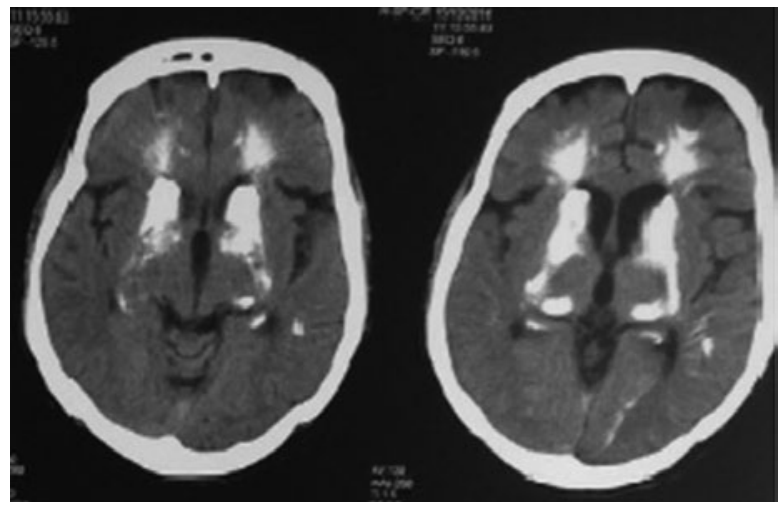

Figura 3. Caso 3. Hipoparatiroidismo. Calcificaciones bilaterales, simétricas en ganglios basales, así como subcorticales.

\section{Algoritmo diagnóstico}

Los exámenes de laboratorio deben incluir niveles de calcio, fósforo y PTH para descartar trastorno paratiroideo.

Si se observa en conjunto con hipodensidad que sugiera enfermedad vascular cerebral, sobre todo en pacientes jóvenes, debe incluirse lactato sérico y líquido cefalorraquídeo, así como biopsia muscular en tinción del Gomori en búsqueda de fibras rojas rasgadas, para descartar enfermedad mitocondrial. En pacientes jóvenes con afectación multiorgánica, sobre todo mujeres, es necesario solicitar anticuerpos antinucleares para descartar neurolupus; si los resultados son negativos, se puede clasificar como trastorno idiopático.

\section{Conclusión}

La calcificación bilateral y simétrica de los ganglios basales es un hallazgo infrecuente que a veces no ocasiona síntomas. Su prevalencia aumenta con la edad y el sitio más afectado es el globo pálido. Las causas más comunes reportadas en la literatura mundial son la enfermedad de Fahr y los trastornos metabólicos del calcio, sin embargo, el diagnóstico diferencial es amplio. Se debe hacer una correlación de hallazgos de imagen típicos con manifestaciones clínicas y resultados de laboratorio, para llegar al diagnóstico definitivo.

\section{Bibliografía}

1. Rumboldt Z, Castillo M, Huang B, Huang B, editores. Brain imaging with MRI and CT: An image pattern approach. EE. UU: Cambridge University Press; 2012.

2. Radaideh AM, Jaradat DM, Haddad FH. Prevalence of incidental basal ganglia calcification on routine brain computed tomography. RMJ. 2012;37(1):6-8.

3. Hathout GM. Clinical Neuroradiology. A case-based approach. Inglaterra: Cambridge University; 2009.

4. Manyam, BV, Walters AS, Narla KR. Bilateral striopallidodentate calcinosis: clinical characteristics of patients seen in a registry. Mov Disord. 2001;16(2):258-264.

5. Benke T, Karner E, Seppi K, Delazer M, Marksteiner J, Donnemiller E. Subacute dementia and imaging correlates in a case of Fahr's disease. J NeurolNeurosurgPsychiatry. 2004;75(8):1163-1165.

6. Eskandary H, Sabba M, Khajejpour F, Eskandari M. Incidental findings in brain computed tomography scans of 3000 head trauma patients Surg Neurol. 2005;63(6):550-553.

7. Daghighi MH, Rezaei V, Zarrintan S, Pourfathi H. Intracranial physiological calcifications in adults on computed tomography in Tabriz, Iran. Folia Morphol (Warsz). 2007;66(2):115-119.

8. Lauterbach EC, Cummings JL, Duffy J, Coffey CE, Kaufer D, Lovell M, et al. Neuropsychiatric correlates and treatment of lenticulostriatal diseases: a review of the literature and overview of research opportunities in Huntington's, Wilson's, and Fahr's diseases.A report of the ANPA Committee on Research. J Neuropsychiatry Clin Neurosci. 1998;10(3):249-266.

9. Johnson JM, Legesse B, Camprodon JA, Murray E, Price BH. The clinical significance of bilateral basal ganglia calcification presenting with mania and delusions. J Neuropsychiatry Clin Neurosci.2013;25(1):68-71.

10. Abe K, Yoshimura H, Tanaka H, Fujita N, Hikita T, Sakoda S. Comparison of conventional and diffusion-weighted MRI and proton MR spectroscopy in patients with mitochondrial encephalomyopathy, lactic acidosis, and stroke-like events. Neuroradiology. 2004;46(2):113-117.

11. Cano A, Romero Al, Bravo F, Vida JM, Espejo S. Síndrome MELAS: hallazgos neurorradiológicos. Radiología. 2002;44(2):69-74.

12. Bianchi MC, Tosetti M, Battini R, Manca ML, Mancuso M, Cioni G, et al. Proton MR spectroscopy of mitochondrial diseases: analysis of brain metabolic abnormalities and their possible diagnostic relevance. Am J Neuroradiol. 2003;24(10):1958-1966.

13. Basak RC. A case report of basal ganglia calcification; a rare finding of hypoparathyroidism. Oman Med J. 2009;24(3):220-222.

14. Wong E, Dahl M. Basal Ganglia Calcification in idiopathic hypoparathyroidism. BCMJ. 2013;55(10):462-465.

15. Rastogi R, Beauchamp NJ, Ladenson PW. Calcification of the basal ganglia in chronic hypoparathyroidism. J Clin Endocrinol Metab. 2003;88(4):1476-1477.

16. Mufaddel AA, Al-Hassani GA.Familial idiopathic basal ganglia calcification (Fahr's disease).Neurosciences (Riyadh). 2014;19(3):171-177.

17. Shahidi GA, Safdarian M. Fahr disease: idiopathic basal ganglia calcification. Iran J Neurol. 2017;16(1):53-54.

18. Contreras $\mathrm{P}$, Elso MJ, Ramírez DC, Cartier L. Pseudo-infarto cerebral como primeramanifestación de un MELAS tardío. Rev Chil Neuro-Psiquiat. 2008;46(1):35-42.

19. Benavente E, Paira S, Roverano S, Saredo G. Encefalitis con estatus convulsivo localizado en una paciente con lupus eritematoso sistémico. Reumatol Clin. 2013;9(2):123-127. 